\title{
Jugular venous ' $a$ ' wave in dilated cardiomyopathy: sign of abbreviated right ventricular filling time
}

\author{
C H Lee, H B Xiao, D G Gibson
}

\begin{abstract}
Objective-To study the mechanisms underlying the high venous pressure often seen in patients with dilated cardiomyopathy.

Design-Retrospective and prospective examination of the pattern of flow in the superior vena cava, cardiac echoDoppler studies, and recordings of the jugular venous pulse.
\end{abstract}

Setting-A tertiary referral cardiac centre.

Patient participants-23 patients with dilated cardiomyopathy, all with functional mitral and tricuspid regurgitation.

Results-Two patterns of venous pulse were seen: a dominant ' $a$ ' wave and ' $x$ ' descent, with systolic flow in the superior vena cava (group $1, n=11$ ), and a dominant ' $v$ ' wave with ' $y$ ' descent and diastolic flow in the superior vena cava (group $2, \mathbf{n}=12$ ). A comparison of group 1 and group 2 showed: age (mean (SD)) 58 (12) $v 61$ (6) years, left ventricular end diastolic dimension $7.0(0.7) \mathrm{cm}$ in both groups, right ventricular short axis $3 \cdot 3$ $(0.6) v 3.6(0.5) \mathrm{cm}$ and long axis $7.3(0.5)$ $v 7 \cdot 1(0.7) \mathrm{cm}$, and duration of tricuspid regurgitation $350(65) v 370(50) \mathrm{ms}$. The RR interval (550 (100) $v 680(80) \mathrm{ms})$ and right ventricular filling time (150 (30) $v$ $290(50) \mathrm{ms}$ ) were significantly shorter in group 1. In all patients in group 2 right ventricular filling time was more than 200 ms with separate $E$ and $A$ waves on the tricuspid Doppler echocardiogram, while in all group 1 patients it was less than $200 \mathrm{~ms}$ with a single summation peak. In nine patients in group 1 , the right ventricular filling time was limited by prolonged tricuspid regurgitation and in the remaining two by prolonged isovolumic relaxation time $(215(80) \mathrm{ms})$, so that it was consistently significantly less than that of the left ventricle.

Conclusion-In patients with dilated cardiomyopathy, right ventricular filling time may be so short that it limits stroke volume. Such patients can be recognised by a dominant ' $a$ ' wave on the jugular venous pulse. Patients in whom the right ventricular filling time was longer showed a dominant ' $v$ ' wave. Both groups can present as "congestive heart failure".
The jugular venous pressure is often raised in patients with dilated cardiomyopathy and this is taken to be a sign of "congestive heart failure" ${ }^{1}$ We found that the characteristics of the venous pulse in these patients varied considerably from patient to patient. In particular, we have been struck by how frequently a dominant ' $a$ ' wave can occur in the absence of severe pulmonary hypertension or organic tricuspid valve disease. Because a dominant ' $a$ ' wave seems to reflect a specific disturbance of right ventricular filling and because its recognition may well modify treatment we examined its underlying mechanism in more detail.

\section{Patients and methods}

STUDY POPULATION

We studied 23 patients ( 18 men and five women, aged 43-84) with dilated cardiomyopathy (defined as a left ventricular end diastolic dimension $>6.5 \mathrm{~cm}$ and fractional shortening less than $20 \%$ ). All the patients were in sinus rhythm and had a raised jugular venous pressure. They were selected only in so far as they fulfilled these criteria and had technically satisfactory records.

\section{MODE AND CROSS SECTIONAL}

ECHOCARDIOGRAPHY

$M$ mode echograms of the mitral valve and left ventricle were recorded with a $3.0 \mathrm{MHz}$ transducer and ATL Imager Mk 300I. The left parasternal long axis view was used with the patient in the left lateral position.

Phonocardiograms and electrocardiograms were recorded simultaneously on a Honeywell recorder at a paper speed of $10 \mathrm{~cm} / \mathrm{s}$. The internal dimension of the left ventricle was measured at end diastole (that is at the onset of QRS complex on the electrocardiogram) and at end systole (at aortic closure) by the leading edge method.

The right ventricle was measured from end diastolic frames of the apical four chamber view. The short axis was measured transversely at the tip of the tricuspid valve leaflets and the long axis from the central point of the tricuspid annulus to the apex.

\section{DOPPLER ECHOCARDIOGRAPHY}

Doppler recordings were made with Doptek equipment and a $2.0 \mathrm{MHz}$ transducer. Regurgitant flow across the tricuspid and mitral 
valves was recorded from the apex, whereas flow in the superior vena cava was measured from the right supraclavicular fossa. Simultaneous phonocardiograms and electrocardiograms were again recorded. The durations of mitral and tricuspid regurgitation were measured for each patient and the interval between two successive regurgitant signals was taken to be the filling time. Measurements were made on three cardiac cycles and the mean value obtained. The flow pattern in the superior vena cava was individually analysed in conjunction with the jugular venous pulse tracings.

JUGULAR VENOUS PULSE RECORDING

This was recorded with the patient in a semirecumbent position. A pressure transducer with a time constant of 4 seconds was used in this study. Simultaneous phonocardiograms and electrocardiograms were recorded. Where possible, the jugular venous pulse traces were superimposed on the Doppler display of flow in the superior vena cava. In patients with a dominant ' $v$ ' wave, the increase in the venous pulse above its minimum value was measured at end diastole and expressed as a percentage of the overall excursion during the cardiac cycle.

STATISTICAL ANALYSIS

Values are given as mean (SD). Differences between mean values were compared by Student's $t$ test.

\section{Results}

JUGULAR VENOUS PULSE AND FLOW IN THE SUPERIOR VENA CAVA

We saw two patterns of venous pulse and flow in the superior vena cava: patients in group 1 $(\mathrm{n}=11)$ had a dominant ' $\mathrm{a}$ ' wave and ' $\mathrm{x}$ ' descent on the jugular venous pulse (fig $1 \mathrm{~A}$ ) and systolic flow in the superior vena cava (fig 1B) whereas those in group $2(\mathrm{n}=12)$ had a dominant jugular venous ' $v$ ' wave and ' $y$ ' descent (fig 2A) and diastolic flow in the superior vena cava (fig 2B). In group 2 the venous pressure rose mainly in diastole $(83(38) \%$ of total excursion) in 10 of the 12 patients, and in the other two, the dominant increase was in systole.

\section{CHARACTERISTICS OF PATIENTS ACCORDING TO} VENOUS PULSE PATTERN (TABLE)

Age, left ventricular end diastolic dimension, ejection fraction, right ventricle to right atrium pressure drop, right ventricular short and long axes, and the duration of tricuspid regurgitation were similar in the two groups of patients.

The RR interval and right ventricular filling time were, however, shorter in group 1 patients $(\mathrm{p}<0.01)$. In all group 2 patients right ventricular filling time was $>200 \mathrm{~ms}$, with $\mathrm{E}$ and $\mathrm{A}$ waves visible on the transtricuspid Doppler flow; whereas all group 1 patients had a right ventricular filling time shorter than $200 \mathrm{~ms}$ and a single summation peak on the tricuspid Doppler flow (fig 3A). Unlike the filling time, there was considerable overlap of $R R$ intervals measured in group 1 and group 2) (group 1,
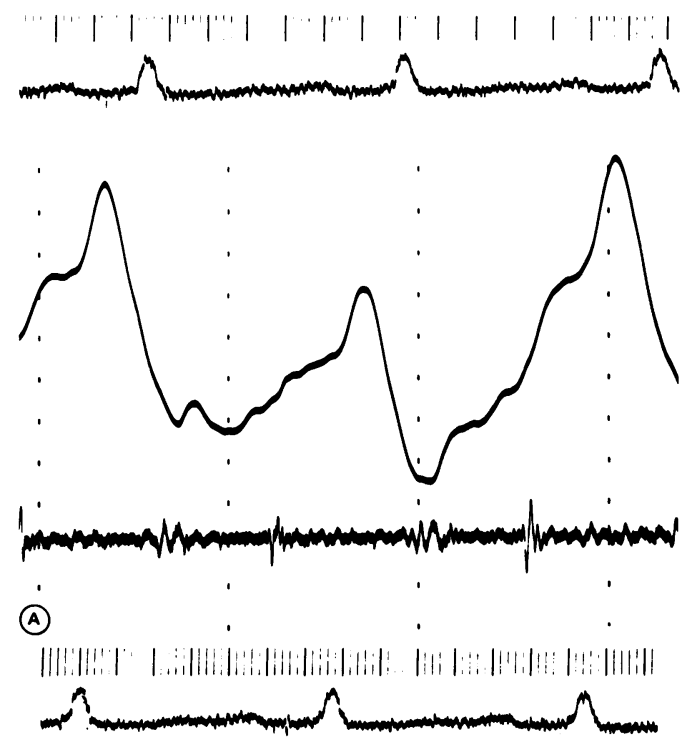

\section{Discussion}

A dominant ' $a$ ' wave in the venous pulse is a striking physical sign and always needs to be explained. We found it in half of our patients 
Figure 2 (A) Jugular venous pulse from another patient with dilated cardiomyopathy showing a dominant ' $v$ ' wave and ' $y$ ' descent. There was no clearly defined ' $a$ ' wave or ' $x$ ' descent. (B) Pulsed Doppler recording of flow velocity in the superior vena cava in the same patient. Downward flow was almost exclusively diastolic and followed the second heart sound on the simultaneous

phonocardiogram. The third heart sound

corresponded to the nadir of the flow velocity signal. (Doppler full scale deflection is $4 \mathrm{kHz}$.)

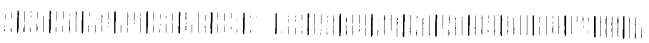

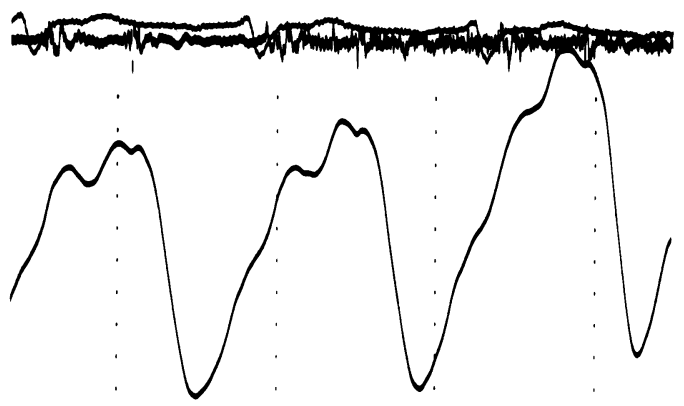

(A)
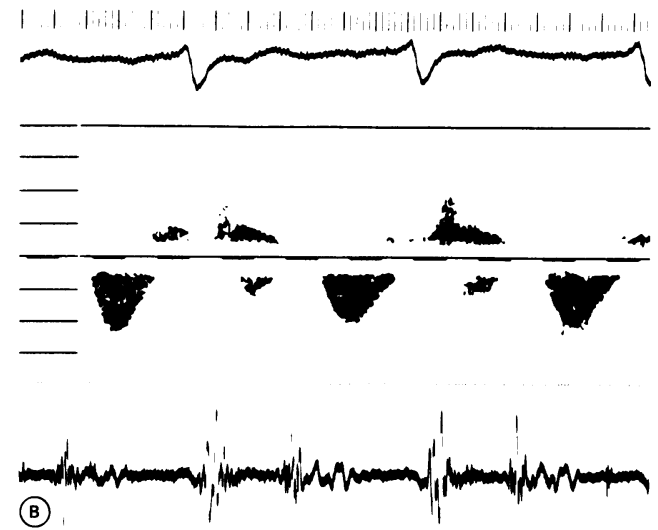

with dilated cardiomyopathy with a raised venous pressure. It seemed surprisingly common in view of how little it has been written about. Though this physical sign was well known to Mackenzie, ${ }^{2}$ it has been overlooked and knowledge of it subsequently lost. It is not mentioned, for example, in any of the standard textbooks of cardiology. ${ }^{3-6}$

Patients with a dominant ' $a$ ' wave and without were indistinguishable in terms of age, left and right ventricular cavity size, or right ventricle to right atrium pressure drop as measured by continuous wave Doppler. There were two major differences: heart rate was higher (109 as against 88 beats per minute) and in particular there was no overlap in right ventricular filling time between the two groups, the mean being $150 \mathrm{~ms}$ in those with dominant ' $\mathrm{a}$ ' wave and $290 \mathrm{~ms}$ in those without. At a rapid heart rate, right ventricular filling time was limited in most patients by the tricuspid regurgitation. Forward flow could not start until the end of the regurgitation, and it was terminated either by the succeeding ventricular systole or (in three patients) prematurely by the onset of diastolic tricuspid regurgitation associated with a prolonged $P R$ interval. The right ventricular filling time was thus shortened and its onset delayed until after the $P$ wave of the succeeding beat. Early diastolic and atrial flow were thus superimposed ("summated") to give a single, short pulse across the tricuspid valve. The venous pulse might therefore be described in the same way, although its timing and duration were characteristic of an 'a' wave.

We have previously described shortened filling time in patients with dilated cardiomyopathy of the left side of the heart. ${ }^{7}$ In patients with functional mitral regurgitation the time available for forward flow is significantly less than that predicted from the period that the mitral valve cusps are separated and is limited to the interval between the pulses of regurgitation. The overall duration of the regurgitation varies little with heart rate, so that as RR interval falls, filling time becomes disproportionately shortened. When filling time drops below $200 \mathrm{~ms}$ the pattern of the flow velocity curve changes; separate $\mathrm{E}$ and $\mathrm{A}$ waves are lost and replaced by a single peak, often accompanied by a summation sound. The short time available for filling thus seems to limit stroke volume, suggesting an analogy with mitral stenosis, though on a temporal rather than a spatial basis. The same mechanisms seemed to be present in our patients on the right side of the heart. In patients with a dominant ' $a$ ' wave, filling time was again less than $200 \mathrm{~ms}$, raising the obvious analogy with temporal tricuspid stenosis. Gibson and Wood comprehensively described the physical signs of rheumatic tricuspid stenosis. ${ }^{8}$ They pointed out that in such patients a clinical diagnosis could be confidently based on a dominant ' $a$ ' wave in the venous pulse and the absence of a deep ' $y$ ' trough or physical signs of severe pulmonary hypertension. These signs are strikingly similar to those found in our patients with dilated cardiomyopathy and they extend the analogy between temporal and spatial atrioventricular valve stenosis.

Several other possible mechanisms might have caused the 'a' wave in our patients. The size of the right ventricular cavity was similar in the two groups of patients. The systolic pressure drop from right ventricle to right atrium was virtually identical irrespective of the pat-

Jugular venous pulse patterns and associated features (mean (SD))

\begin{tabular}{|c|c|c|c|}
\hline Variable & $\begin{array}{l}\text { Group } 1 \\
\text { Dominant ' } a \text { ' wave } \\
(n=11)\end{array}$ & $\begin{array}{l}\text { Group } 2 \\
\text { Dominant ' } v \text { ' wave } \\
(n=12)\end{array}$ & p Values \\
\hline $\begin{array}{l}\text { Age (yr) } \\
\text { RR intervals (ms) } \\
\text { Left ventricular end diastolic dimension }(\mathrm{cm}) \\
\text { Right ventricular short axis }(\mathrm{cm}) \\
\text { Right ventricular long axis }(\mathrm{cm}) \\
\text { Left ventricular end systolic dimension }(\mathrm{cm}) \\
\text { Ejection fraction }(\%) \\
\text { Right ventricle-right atrium pressure drop }(\mathrm{mm} \mathrm{Hg}) \\
\text { Duration of tricuspid regurgitation }(\mathrm{ms}) \\
\text { Right ventricular filling time }(\mathrm{ms}) \\
\text { Duration of mitral regurgitation }(\mathrm{ms}) \\
\text { Left ventricular filling time }(\mathrm{ms})\end{array}$ & $\begin{array}{l}58(12) \\
550(100) \\
7 \cdot 0(0 \cdot 7) \\
3 \cdot 3(0 \cdot 6) \\
7 \cdot 3(0 \cdot 5) \\
6 \cdot 3(0 \cdot 8) \\
12(5 \cdot 4) \\
30(5) \\
350(65) \\
150(30) \\
340(40) \\
215(80)\end{array}$ & $\begin{array}{l}61(6) \\
680(80) \\
7 \cdot 0(0 \cdot 7) \\
3 \cdot 6(0 \cdot 5) \\
7 \cdot 1(0 \cdot 7) \\
6 \cdot 0(0 \cdot 7) \\
14(3 \cdot 9) \\
30(5) \\
370(50) \\
290(50) \\
340(30) \\
335(60)\end{array}$ & $\begin{array}{l}\text { NS } \\
<0.01 \\
\text { NS } \\
\text { NS } \\
\text { NS } \\
\text { NS } \\
\text { NS } \\
\text { NS } \\
\text { NS } \\
<0.01 \\
\text { NS } \\
<0.01\end{array}$ \\
\hline
\end{tabular}


Figure 3 (A) Pulsed Doppler recording of forward tricuspid flow velocity. Overall filling time was reduced to $80 \mathrm{~ms}$ and occurred exclusively after the $P$ wave of the succeeding beat. (Doppler full scale deflection is $4 \mathrm{kHz}$.) (B) Continuous wave Doppler recording showing tricuspid regurgitation signal from the same patient. PR interval was prolonged and the onset of the regurgitation was presystolic. The interval between the pulses of regurgitation corresponded to the short right ventricular filling time. (Doppler full scale deflection is $16 \mathrm{kHz}$.)

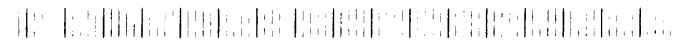

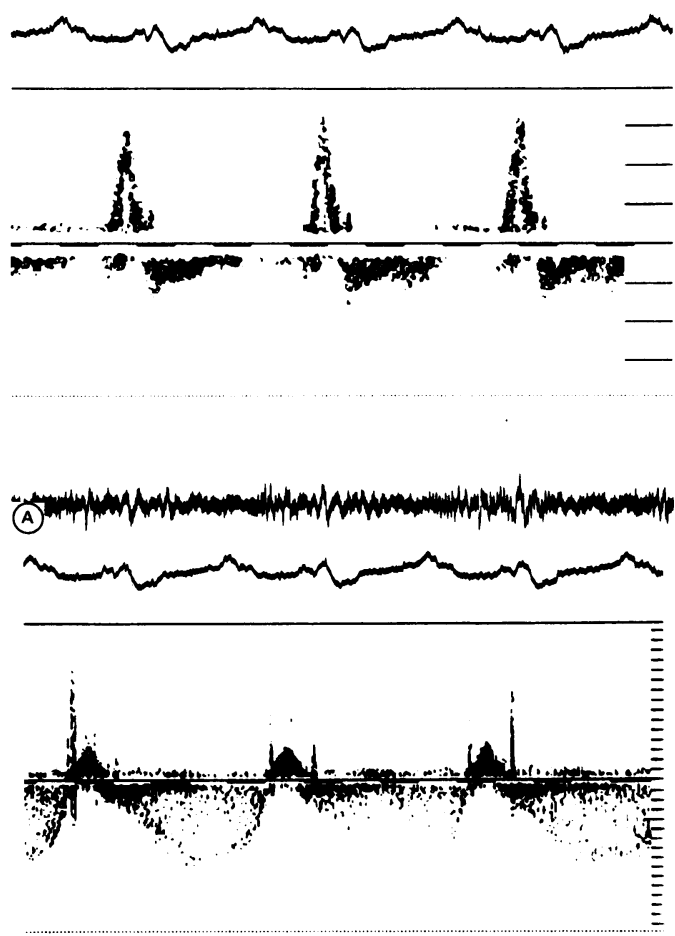

m.m.

Bi||||||||||||||||||||||||||||||||||||||||||||||||||||||||||||||||||||||||||||||

tern of the venous pulse. This shows that the severity of pulmonary hypertension did not account for the dominant ' $a$ ' wave. Right ventricular diastolic function might have been impaired by primary disease or as the result of Bernheim effect from the left ventricle. ${ }^{9}$ In patients whose heart rates were slower this dysfunction manifested itself in the characteristic way as a deep ' $y$ ' descent and dominant increase in venous pressure during diastole. A pressure increase that occurred predominantly during systole, as would be expected if tricuspid regurgitation were the mechanism, was much less frequent. This makes it unlikely that the tricuspid regurgitation was haemodynamically significant or that differences in the severity of regurgitation explained our findings. We conclude, therefore, that a dominant ' $a$ ' wave in the venous pulse in patients with dilated cardiomyopathy is not caused by primary right ventricular disease, severe pulmonary hypertension, or impaired diastolic function as the result of left side involvement, but is the direct effect of the limited time available for tricuspid inflow. "Temporal tricuspid stenosis" was therefore present in about half of our patients.

When the venous pressure is raised in patients with dilated cardiomyopathy, they are often said to be in "congestive heart failure". Though the term has never been adequately defined, heart failure is usually regarded as an undesirable state of affairs that should be treated. The standard treatment for a raised venous pressure is to administer diuretics until it falls. When filling time is limited, a normal stroke volume can only enter the ventricle if mean flow rate is increased; this requires an increase in acceleration rate at the start of filling and deceleration rate at its end. Such velocity changes require corresponding pressure gradients along the direction of flow of approximately $1 \mathrm{~mm} \mathrm{Hg} / \mathrm{cm}$ for each $1 \mathrm{~g}$ of acceleration. The pressure wave recorded in the venous pulse directly reflects the pressure changes across the valve that make this rapid filling possible. To lower venous pressure would thus be to remove a potential compensatory mechanism. It seems better either to reduce the duration of the tricuspid regurgitation or to lower the heart rate, both of which would increase filling time. This approach to treatment is quite different from that to be adopted if the venous pressure is raised by functional tricuspid regurgitation or reduced ventricular compliance. Our results show yet again that many different pathological mechanisms may be responsible for "heart failure"-a clinical diagnosis that is imprecise. It is quite possible with simple clinical skills to recognise the disturbance we have described and it can readily be confirmed non-invasively. More effective treatment is then likely to follow from more precise diagnosis.

HBX is supported by the Brompton Hospital Special Cardiac Fund.

1 Fishberg AM. The venous pressure and pulse. In: Heart failure. Philadelphia: Lea and Febiger, 1940:96-119.

2 Mackenzie J. The auricular form of the venous pulse. In: The study of the pulse: arterial, venous and hepatic, and the movement of the heart. Edinburgh: Pentland, 1902: 197-208.

3 Wenger NK, Goodwin JF, Roberts WC. Cardiomyopathy and myocardial involvement in systemic disease. In: Hurst JW, ed. The heart, arteries and veins. New York: McGrawHill, 1986:1181-248.

4 Shabetai R. Dilated cardiomyopathy. In: Parmley WW, Chatterjee K, eds. Cardiology. Philadelphia: Lippincott, 1987:41:1-16.

5 Littler WA. Dilated cardiomyopathy. In: Julian DG, Camm AJ, Fox KM, Hall RJC, Poole-Wilson PA, eds. Diseases of the heart. London: Bailliere Tindall, 1989:916-24.

the heart. London: Bailliere Tindall, 1989:916-24.
6 Wynne J, Braunwald E. The cardiomyopathies and myocarditides. In: Braunwald E, ed. Heart disease, a textbook of ditides. In: Braunwald E, ed. Heart disease, a textbook of
cardiovascular medicine. Philadelphia: W B Saunders, cardiovascular

$7 \mathrm{Ng} \mathrm{KSK}$, Gibson DG. Impairment of diastolic function by shortened filling period in severe left ventricular disease. Br Heart J 1989;62:246-52.

8 Gibson $\mathrm{R}$, Wood $\mathrm{P}$. The diagnosis of tricuspid stenosis. $\mathrm{Br}$ Heart J 1955;17:552-62.

9 Bernheim DR. De l'asystolie veineuse dans l'hypertrophie du coeur gauche. Rev de Méd 1910;30:785-801. 